\title{
HIGH AND LOW DIABETES INCIDENCE NONOBESE DIABETIC (NOD) MICE: ORIGINS AND CHARACTERISATION
}

\author{
ALAN G. BAXTER, MARIA KOULMANDA and THOMAS E. MANDEL' \\ Walter \& Eliza Hall Institute of Medical Research, Parkville 3050. Australia
}

(Received June 4, 1990; in final form August 24, 1990)

\begin{abstract}
The Nonobese Diabetic mouse (NOD mouse) is an established model of autoimmune diabetes mellitus. While all colonies of NOD mice are derived from a single diabetic female detected during the breeding of a cataract-prone strain of mice, some of the dispersed colonies have been separated for many generations and express varying levels of diabetes. It is unciear to what extent this is due to environmental factors such as diet factor or a result of the varied origins of the colonies. Here we compare the incidence of diabetes and severity of insulitis in two divergent lines of NOD mice that differ in incidence of disease, but are maintained in the same environment. Fl crosses were performed and the progeny found to express the disease incidence of the low incidence line. This finding is consistent with either a dominant resistance gene(s) being responsible for reduced penetrance of disease or a transmissible environmental agent reducing the severity of the autoimmune process.
\end{abstract}

KEY WORDS: Diabetes mellitus, NOD, nonobese diabetic mouse, breeding, mixed lymphocyte culture

The NOD mouse was derived from JCL:ICR outbred mice during the derivation of the cataract prone CST strain'. After 6 generations of brother/ sister mating, two sublines of mice were separated on the basis of fasting blood sugars. After 20 generations of brother/sister mating, a single female in the normoglycemic line was noted to be of low weight, polyuric, polydipsic and glycosuric. The NOD strain was derived from this female ${ }^{1.2}$. Demand for NOD mice was great, and a production colony was established at Shionogi. This colony was not brother/ sister mated, but random mated. It is not clear at what generation this colony was established, but it is likely to have been at least a further 20 generations of brother/sister mating after the discovery of the index female. At some time around 1984, it was noticed that the cumulative incidence of diabetes in mice from the Shionogi production colony was considerably less than that of NOD mice derived from the breeding nucleus maintained by Prof S. Makino (Personal communication, Dr M Hattori). The colony maintained by $\mathrm{Dr} \mathrm{H}$. Asamoto at the Kyoto National Hospital, Kyoto, Japan was derived from the random mated production colony during this period. The NOD/ Wehi line of mice was founded from breeding pairs kindly donated by $\operatorname{Dr~H}$. Asamoto in May 1984 (Figure 1) and express a lower cumulative incidence of diabetes than most other colonies of NOD mice.

\footnotetext{
To whom all correspondence should be addressed.
}

In $1985 \mathrm{Dr} \mathrm{M}$. Hattori brought breeding pairs obtained from the breeding nucleus maintained by Prof S. Makino in Shionogi to the USA. The line derived from these mice is currently maintained at the Joslin Diabetes Center in Boston and by Dr E.H. Leiter at the Jackson Laboratory, Bar Harbor, Maine. In both Massachusetts and Maine, these mice maintain a high incidence of disease with $85-95 \%$ of female mice becoming diabetic before 210 days of age $^{3}$. The NOD/Lt line maintained at the Hall Institute was kindly provided by Dr E.H. Leiter in January 1987, and maintains the same incidence of diabetes as the parental colony.

The NOD/Wehi and NOD/Lt mice are bred in separate halves of the Hall Institute's specific pathogen free (SPF) breeding facility. At 3-6 weeks of age they are transferred to SPF animal rooms within the laboratory complex where they are housed on the same rack in the same room. Both lines receive the same food (Barastock Mouse Pellets, Vic) and water (filtered, acidified). We have previously reported that these two lines accept reciprocal skin grafts, fail to react to each other in mixed lymphocyte culture (MLC), and share 16 isozymes ${ }^{4}$. Minisattelite restriction fragment polymorphisms have not been found between the lines. The only difference, other than disease incidence, found thus far is that NOD/Wehi mice have an increased syngeneic mixed lymphocyte response compared to NOD/Lt and BALB/cAnBradley Wehi mice. The syngeneic response of NOD/Lt 


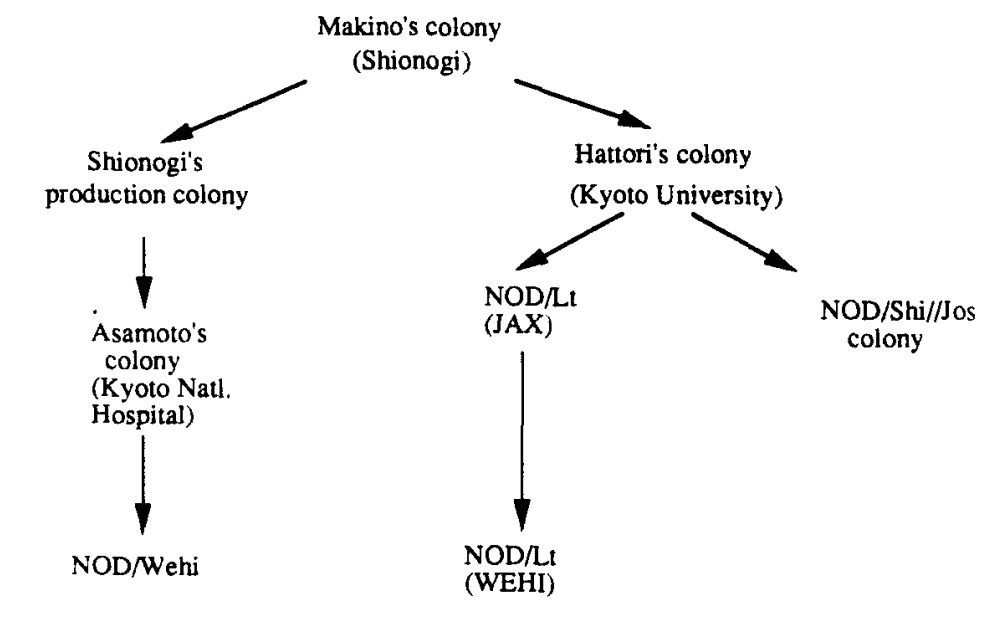

Figure 1 The origins or the NOD/Lt and NOR/Wehi lines of Nonobese Diabetic Mice maintained at WEHI. Source: Dr M Hattori.

nylon wool passaged splenic $T$ cells averaged $5 \%$ $(S D=5, n=6)$ of the allogeneic response to CBA/ CaHWehi irradiated splenocytes, while the syngeneic response of NOD/Wehi splenic $T$ cells was $19 \%$ $(S D=3, n=6)$ of the allogeneic response. The allogeneic response was similar in both lines.

In this study, we confirmed the difference in cumulative incidences of diabetes in NOD/Lt and NOD/Wehi mice and compared the severity of insulitis to test the hypothesis that this difference could be attributed to immunological factors. Three possible mechanisms of transfer are proposed to explain the heritable resistance to diabetes in the NOD/Wehi line: Genetics. vertical passage of a protective pathogen, and mitochondrial transmission of a protective factor. F1 mice derived from interbreeding the two lines were studied to test these possibilities.

\section{MATERIALS AND METHODS}

Mice

NOD/Wehi mice were obtained from the colony maintained at the Walter \& Eliza Hall Institute (WEHI) for 21 generation and NOD/Lt mice were obtained from the colony maintained at the WEHI for 9 generations. All mice were housed under specific pathogen free conditions and were allowed free access to food (Barastock mouse pellets, VIC.) and acidified water.

\section{Breeding}

The two parental lines are maintained by brother/ sister mating. The mice selected to produce the crosses were randomly selected from litters born of nucleus breeders in the parental lines.

\section{Serum Glucose Measurement}

Each mouse was individually tagged and bled of $20-$ $40 \mu \mathrm{l}$ at two weekly intervals and the serum glucose concentration measured by the glucose oxidase technique with a Beckman Glucose Analyzer (Beckman, USA). A mouse was declared diabetic if it was found to have a random serum glucose level greater than $12 \mathrm{mMol} / 1$ followed by either rapid demise or further values greater than $12 \mathrm{mMol} / 1$ on subsequent bleeds. Mice found dead without a previous raised serum glucose measurement were excluded from analysis.

\section{Histological Assessment of Insulitis}

Pancreata were collected from most of the mice which had been followed by serial bleeding when the mice were: recently deceased and not autolysed, demonstrated to be hyperglycemic for 3 or more glucose estimations, or older than 250 days. The whole pancreas was fixed in Bouin's fluid, transferred to alcohol, paraffin embedded and sectioned at three levels. Sections were stained with hematoxylin and eosin or Gomori aldehyde fuchsin, and independently scored by two observers. A semi-objective score from 0-4 was assigned to each islet seen. A score of 0 indicated an islet free of pathology, and a score of 4 indicated virtually complete destruction. The scores were then totalled and expressed as a percentage of the total possible score. 
Table 1 Both lines of NOD mice were tested for serological evidence of infection at 3 month intervals. The following specificities were not found:

\section{Ectromelia}

Murine Hepatitis Virus

Mouse Polyoma

Sendai Virus

Reovirus 3

Mouse Adenovirus

Theller's GD-VII Virus

Lymphocytic Choriomenigitis Virus

Minute Virus

Rotavirus

EMC

Chlamidia

Mycoplasma

\section{Statistical Analysis}

Comparison of cumulative incidence of diabetes was made using the $\chi^{2}$ test. It was assumed that the insulitis scores of pancreata maintained the same (not necessarily normal) distribution and the Rank Sum Test
Table 2 Cumulative incidence of diabetes in males and females of the NOD/Lt and NOD/Wehi lines at 150 and 250 days. Statistical comparison was performed with the Chi Squared test.

\begin{tabular}{lcc}
\hline Line & $150 d$ & $250 d$ \\
\hline NOD/Lt females & $23 / 50(46 \%)$ & $40 / 44(91 \%)$ \\
NOD/Wehi females & $7 / 50(14 \%)$ & $21 / 45(47 \%)$ \\
Significance & $p<0.005$ & $p<0.0001$ \\
NOD/Lt males & $2 / 48(4 \%)$ & $10 / 47(21 \%)$ \\
NOD/Wehi males & $1 / 50(2 \%)$ & $3 / 50(6 \%)$ \\
Significance & NS & $p<0.05$ \\
\hline
\end{tabular}

was applied. The age at onset of diabetes was compared by assuming a normal distribution and applying the students' $t$ test.

\section{RESULTS}

\section{Confirmation of Specific Pathogen Free Conditions}

Pathogen serology was performed 3 monthly by the Veterinary Laboratories of the Department of Agri-

\section{(a)}

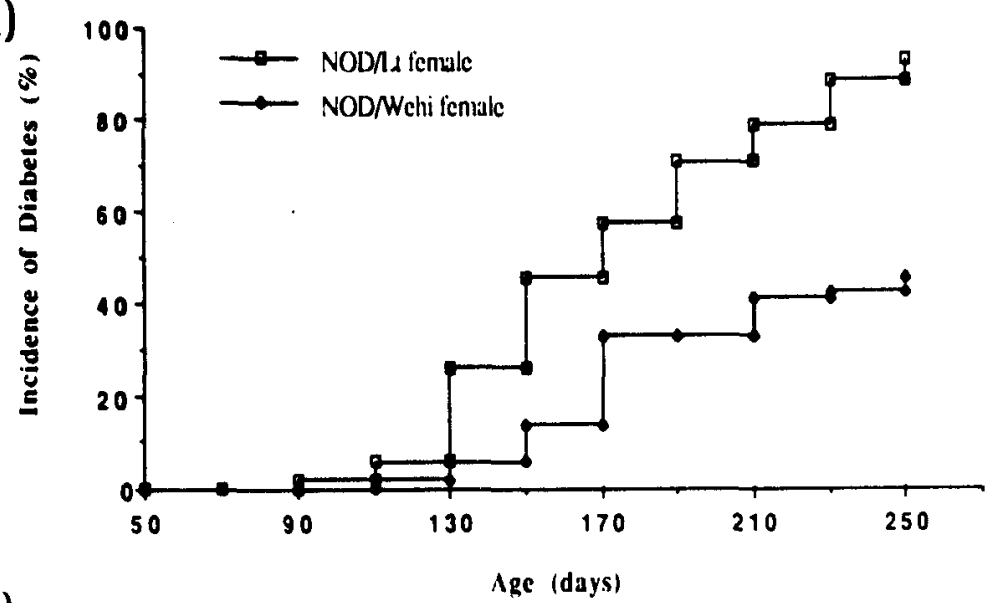

(b)

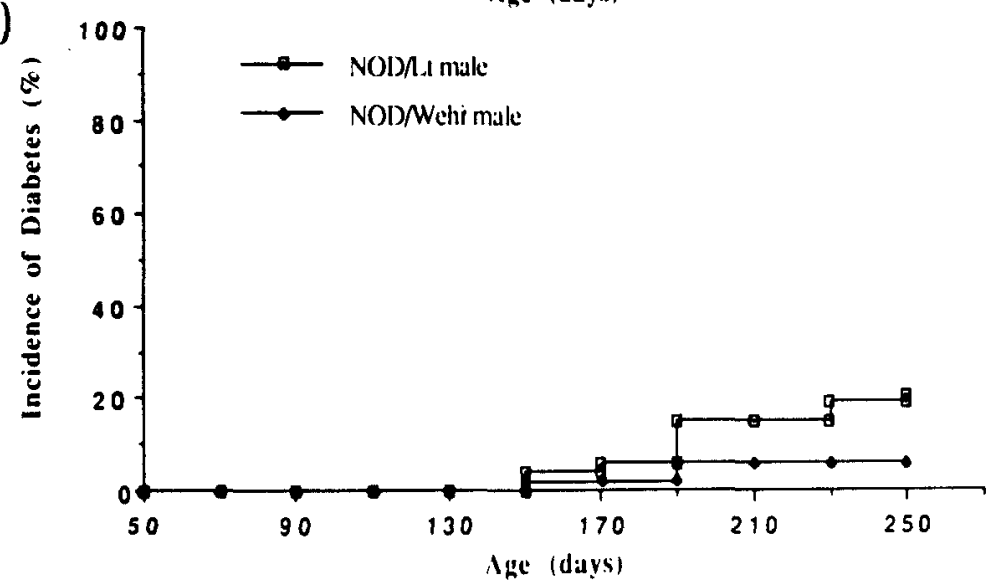

Figure 2 Proportion of NOD/Lt and NOD/Wehi female mice to develop diabetes betore 250 days of age. Diabetes was defined as random serum glucose greater than $12 \mathrm{mMol} / 1$ followed by either rapid demise or further raised glucose estimations. Serum glucose estimations were performed at two-weekly intervals. 
culture (Adelaide, South Australia) on multiple samples of pooled sera. The serological specificities tested are listed in Table 1 . None of these were positive. Coagulase positive Staphylococcus aureus has been isolated from housing furniture and cultured from abscesses in both lines.

\section{Incidence of Diabetes in NOD/Lt and NOD/Wehi Lines}

The NOD/Lt female mice become diabetic earlier and at a greater rate than NOD/Wehi female mice (Figure $2 a)$. The lines maintain a highly significant difference in cumulative incidence of diabetes in females at both 150 and 250 days (Table 2). Both lines express a much lower incidence of disease in males. The difference in disease incidence in males only achieves significance at 250 days (Figure $2 b$ ). The NOD/Lt female mice that became diabetic did so at a mean age of 150 days (standard deviation 37 days), while the NOD/Wehi female mice became diabetic at a mean of 163 days (standard deviation 35 days, not significant, students $t$ test). The NOD/Lt male mice that became diabetic did so at a mean age of 202 days (standard deviation 42 days), while the NOD/Wehi males became diabetic at a mean of 162 days (standard deviation 19 days (not significant, students' $t$ test). A second cohort of 100 NOD/Wehi mice (50 male, 50 female) were subsequently followed to assess variation in incidence of diabetes with time. The second cohort varied by less than $6 \%$ from the values obtained from the initial study (not significant, $\chi^{2}$ test; Table 4 ).

\section{Histological Examination of the Pancreata of NOD Lines}

All diabetic mice examined were found to have insulitis scores in excess of $95 \%$. A significant difference was found between the insulitis scores of both the female $(p<0.01)$ and male $(p<0.02)$ NOD/ $\mathrm{Lt}$ and NOD/Wehi mice (Figure 3 ). As some pancreata of diabetic animals which died unexpectedly were not obtained. there is some bias inherent in the collection of this data. To assess the effect of this bias, the data were re-analysed assuming an insulitis score of $100 \%$ for all animals known to be diabetic at the

Table 3 Incidence of diabetes in $\mathrm{Fl}$ crosses between NOD/Lt and NOD/Wehi lines. The direction of the mating does not significantly affect the incidence of diabetes ( $x^{2}$ test)

\begin{tabular}{lllc}
\hline CROSS & SEX & $150 d$ & $250 d$ \\
\hline (NOD/LT X NOD/Wehi)FI & $\mathrm{F}$ & $7 / 18(39 \%)$ & $9 / 18(50 \%)$ \\
& $\mathrm{M}$ & $1 / 22(5 \%)$ & $2 / 21(10 \%)$ \\
& & & \\
(NOD/Wehi X NOD/Li)FI & $\mathrm{F}$ & $4 / 19(21 \%)$ & $10 / 19(53 \%)$ \\
& $\mathrm{M}$ & $1 / 25(4 \%)$ & $1 / 23(4 \%)$ \\
\hline
\end{tabular}

Table 4 Incidence of diabetes in F1 crosses between NOD/Lt and NOD/Wehi lines compared with data from a second cohort of NOD/Wehi mice which were followed longitudinally at the same time. and the group of NOD/Lt mice from Table 2. The groups were compared with the $\chi^{2}$ test.

\begin{tabular}{llll}
\hline CROSS & SEX & $150 d$ & $250 d$ \\
\hline F1 Totals & F & $11 / 37(30 \%)$ & $19 / 37(51 \%)$ \\
NOD/Lt & F & $23 / 50(46 \%$, NS) & $40 / 44(91 \%, \mathrm{p}<0.0001)$ \\
NOD/Wehi & F & $14 / 49(29 \%, \mathrm{NS})$ & $25 / 49(51 \%, \mathrm{NS})$ \\
& & $2 / 47(4 \%)$ & $3 / 44(7 \%)$ \\
F1 Totals & $\mathrm{M}$ & $2 / 48(4 \%, \mathrm{NS})$ & $10 / 47(21 \%, \mathrm{p}<0.05)$ \\
NOD/Lt & $\mathrm{M}$ & $2 / 48)$ & $3 / 50(6 \%, \mathrm{NS})$ \\
NOD/Wehi & $\mathrm{M}$ & $0 / 50(0 \%, \mathrm{NS})$ & \\
\hline
\end{tabular}

time of death, but for which histology was not available. This procedure did not affect the results.

\section{Incidence of Diabetes in Crosses Between NOD Lines}

18 female and 22 male (NOD/Wehi X NOD/Lt)F1 and 19 female and 25 male (NOD/Lt X NOD/ Wehi)Fl were observed for 250 days. The direction of the cross did not significantly affect the incidence of diabetes $\left(\gamma^{2}\right.$ test, Table 3$)$. At 150 days, the incidence of diabetes in Fl females was not significantly different from either NOD/Wehi or NOD/Lt females of the same age. By 250 days, however, the F1 females had an incidence very significantly different from NOD/Lt females $(p<0.0001)$, while not significantly different from NOD/Wehi females of the same age. The $F 1$ males also showed a significant difference from NOD/Lt males at 250 days ( $p<0.05, \gamma^{2}$ test, Table 4 ).

\section{Histological Examination of the Pancreata of FI Mice}

The insulitis scores of the $\mathrm{F} 1$ mice were compared with NOD/Wehi and NOD/Lt mice of the same sex by applying the Mann Whitney $U$ (Wilcoxon twosample, or rank sum) test. The $F 1$ females were not significantly different from the NOD/Wehi females but differed from the NOD/Lt females $(p<0.05)$. The $F 1$ males were not found to be significantly different from either the NOD/Wehi or NOD/Lt males (Figure 3).

\section{DISCUSSION}

While the NOD/Lt line maintains a high incidence of diabetes similar to that expressed in its parental colony in Maine, the NOD/Wehi line has an unusually low incidence of diabetes, and this incidence appears to be quite stable as $260 \mathrm{NOD} /$ Wehi mice have now been followed in 3 cohorts by two-weekly serum glucose estimations over 30 months without a change in incidence of diabetes (data from third cohort not 


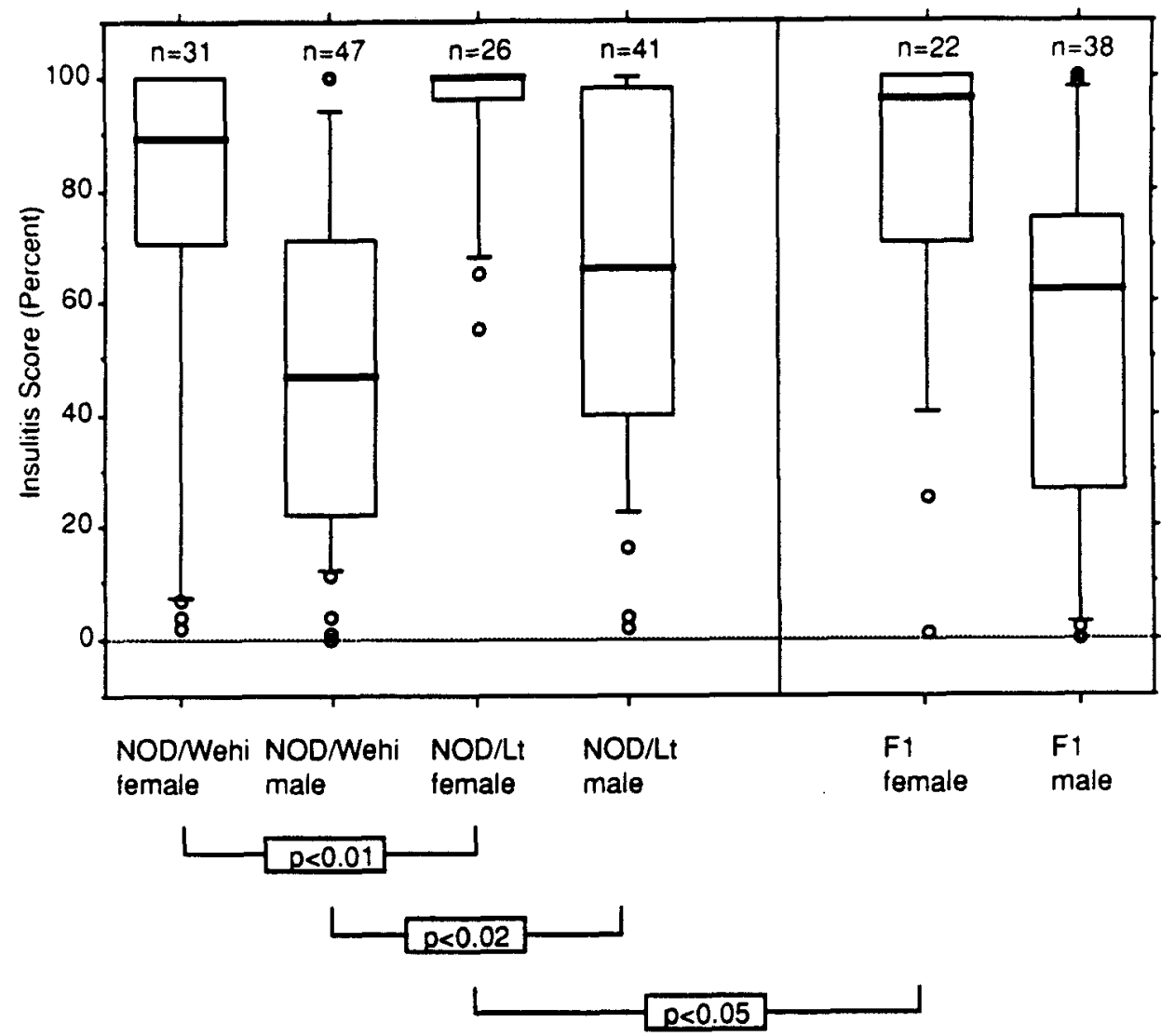

Figure 3 Box plots of insulitis scores on NOD mice aged 275-345d. Pancreata were collected and scored for intensity of insulitis. A semi-objective score from 0-4 was assigned to each islet in all levels. A score of 0 indicated an islet free of pathology, and a score of 4 indicated virtually complete destruction. The scores were then totalled and expressed as a percentage of the total possible score. The box plots represent the insulitis scores of each group of mice. For each group, the heavy line indicates the median; the confines of the box represents the 25 th and 75 th percentiles; the stem represents the 10 th and 90 th percentiles; and the points display outlying values. Groups of the same sex were compared using the Mann-Whitney $U$ (rank sum) test and significant differences indicated by bars below the figure. Fl females were not significantly different from NOD/Wehi females but were different from NOD/Lt females. Fl males were not significantly different from either NOD/Wehi or NOD/Lt males.

shown). Although initial reports described an even lower incidence of disease, this may have been the result of sampling errors given the small numbers of mice availabe at that time.

Three possible mechanisms can be postulated to explain the different incidences of diabetes in the NOD/Wehi and NOD/Lt lines of diabetes prone mice. The first mechanism is genetic; either acquired mutation(s) in the NOD/Wehi that reduce penetrance of the NOD diabetes susceptibility genes, or the absence of one or more of the susceptibility genes normally present in other NOD lines. The second possible mechanism is mitochondrial transmission of a factor resposible for modulating disease incidence. The third possible mechanism is the restricted passage of a pathogen within either line that (in the case of NOD/Wehi) confers resistance to diabetes or (in the case of NOD/Lt) precipitates diabetes.
Due to the early difficulty of obtaining NOD mice, many lines such as the NOD/Wehi were founded from breeding pairs not directly derived from the original breeding nucleus. These lines have since been maintained under standard breding conditions, and some have now been inbred for more than 20 generations, effectively freezing any pre-existing differences and new mutations. The data presented here is consistent with the possibility that the NOD/Wehi line differs genetically from the NOD/Lt line. As the pattern of diabetes in the FI mice is unlike that of the NOD/Lt line, but not significantly different from the NOD/Wehi, this difference would have to be active in the heterozygous state. It may represent one or more dominant resistance genes in the NOD/Wehi or the absence of a recessive diabetes gene which is present in NOD/Lt and acts to increase the penetrance of the other susceptibility genes. These pos- 
sibilities remain to be tested by further breeding experiments.

Mitochondrial transmission of an element responsible for modulating expression of disease would result in transmission of resistance or susceptibility to diabetes from mother to offspring. This would result in a different incidence of diabetes in the Fl's depending on the direction of the cross. That is, that the offspring of an NOD/Wehi mother would express a lower incidence of diabetes than the offspring of an NOD/Lt mother. The data presented here eliminates the possiblility of mitochondrial transmission as the direction of the cross does not significantly affect the incidence of diabetes in the $\mathrm{Fl}$ offspring.

The important role of diet in modulating the penetrance of diabetogenic genes has been demonstrated. Casein ${ }^{5.6}$ and Brewers yeast ${ }^{7}$ appear to increase the incidence of diabetes, while semi-purified or elemental diets reduce it. Unfortunately, most breeding facilities have little or no control over the extract composition of their mouse chow. Thus there is a known uncontrolled variable preventing the meaningful comparison of disease incidence in most NOD colonies. Furthermore, seasonal changes in chow composition may make the use of historical controls within an institution inappropriate. In this study, all animals were fed the same closed formulation chow. The NOD/Lt and NOD/Wehi lines were bled concurrently. The Fl's were followed subsequently, but in parallel with a second cohort of 100 NOD/Wehi mice in which the incidence of diabetes varied by less than $6 \%$ from the values obtained from the initial study. It is therefore unlikely that diet has any part in maintaining the differences between the two lines. Other gross environmental differences are also unlikely as the lines are bred in the same facilities and maintained in the same room.

Immune modulation by viruses has been proposed as a mechanism to both decrease ${ }^{8}$ and increase ${ }^{4}$ the incidence of autoimmune diabetes. The evidence that environmental pathogens may actually protect from diabetes largely comes from the observation of colonies derived from a single source established under varying standards of cleanliness. Generally, a higher incidence of diabetes is found in colonies known to be SPF ${ }^{8}$. This is supported by the finding that transferring SPF NOD mice with an incidence of diabetes of $6 \%$ to germ-free conditions resulted in an increase in the incidence of disease to $71 \%{ }^{10}$. Although protection from diabetes has been demonstrated with the Armstrong 53b strain. clone 13 of lymphocytic choriomeningitis virus (LCM) in both NOD mice" and $\mathrm{BB}$ rats $^{12}$, it is difficult to know how to interpret this in view of the difficulty in reproducing the finding ${ }^{16}$ and the immunosuppressive nature of infection with LCM. The suggestion that viral infection exacerbates autoimmune beta cell destruc- tion in the NOD mouse is almost unsupported by data. An analogy has been drawn between the mild immunosuppressive effects of viral infections and the finding that cyclophosphamide can paradoxically precipitate diabetes in NOD mice $e^{13: 14.15}$.

Although our facilities are SFP and neither line was found to have serological evidence of viral infection. it remains a possibility that restricted passage of a pathogen could be responsible for modulating the expression of disease. The finding that crosses between the NOD/Wehi and NOD/Lt lines express an incidence of disease that significantly differs from that of NOD/Lt but not NOD/Wehi eliminates as a possiblility the restricted passage of a diabetogenic pathogen in the NOD/Lt line, but is still consistent with the restricted passage of a protective pathogen in NOD/Wehi. Such a mechanism suggests very exciting possibilities for the prevention of diabetes in humans. but remains to be tested by serum transfer and mixed boxing experiments.

\section{Acknowledgements}

This work was generously supported by Hoechst Australia Pty Lid., National Health \& Medical Research Council, JB Were and Sons, The Warman Fund and The Perpetual Trustees and Executors Association of Australia. AGB receives a Hoechst postgraduate research scholarship. We would like to thank T. Johns, A. Szalai, S. Hambly, B. Beale and R. Hodgeson for assistance with animal care; Dr C. Guest for advice on data analysis; Dr M. Hattori for encouragement and advice; and Dr E.H. Leiter for his suggestions. Much of the background information on the two strains was kindly provided by $\operatorname{Dr} M$. Hattori.

\section{References}

1. Makino S, Kunimoto K, Muraoka Y. Mizushima Y. Katagiri $\mathrm{K}$. Tochino $\mathrm{Y}$. Breeding of a nonobese diabetic strain of mice Exp Anim 1980; 29: 1-13

2. Ikegami H. Makino S. Harada M. Eisenbarth GS, Hattori M. The cataract Shionogi mouse: Similar class II but different class I gene products. Diaberologia 1988: 31: 254-258

3. Colony Information Sheets. NOD Mouse Workshop. Scottsdale. Arizona. 1989

4. Baxter AG. Adams MA, Mandel TE. Comparison of highand low-diabetes incidence NOD mouse strains. Diahetes 1989: 38: 1296-1300

5. Elliott RB, Reddy SN, Bibby NJ, Kida K. Dietary prevention of diabetes in the non-obese diabetic mouse. Diabetologia 1988; 31: 62-64

6. Scott FW. Daneman D, Martin JM. Evidence for a critical role of diet in the development of insulin-dependent diabetes mellitus. Diabetes Res 1988: 7: 153-157

7. Coleman DL. Kuzava JE. Leiter EH. Effect of diel on the incidence of diabetes in non-obesc diabetic (NOD) micc. Diaheres 1990: in press

8. Leiter EH. The role of environmental factors in modulating insulin dependent diabetes. Symposium Paper. 14th 
Argenteuil Symposium, Belgium, in The role of microorganisms in non-infectious disease. Springer Verlag, Brussels. 1990

9. Mandel TE, Cuthberson RA. Low-dose immunosuppression may unmask type I diabetes mellitus by specific inactivation of suppressor cells. Med Hypotheses 1986; 19: 325-331

10. Suzuki T, Yamada T, Takao T, Fujimura T, Kawamura E, Shimizu ZM, Tamashita R, Nomoto K. Diabetogenic effects of lymphocyte transfusion on the NOD or NOD nude mouse. In: Immune-deficient animals in hiomedical research. 1987; Rygaard J, graem NBN, Sprang-Thomsen M (eds). Karger. Basel

11. Oldstone MBA. Prevention of type 1 diabetes in nonobese diabetic mice by virus infection Science 1988; 239: 500-502

12. Schwimmbeck PL, Dyrberg T, Oldstone MBA. Abrogation of diabetes in BB rats by acute virus infection. $J$ Immunol 1988; 140: $3394-3400$
13. Charlton B, Bacelj A, Slattery RM, Mandel TE. Cyclophosphamide induced diabetes in NOD/Wehi mice: evidence for suppression in spontaneous autoimmune diabetes mellitus. Diabetes 1989; 38: 441-447

14. Harada M, Makino S. Promotion of spontaneous diabetes in non-obese diabetes-prone mice by cyclophosphamide. Diabetologia 1984: 27: 604-606

15. Yasunami R, Bach J-F. Anti-suppressor effect of cyclophosphamide on the development of spontaneous diabetes in NOD mice. Eur J Immunol 1988; 18: 481-484

16. Oldstone MBA, Ahmed R, Salvato $M$. Viruses as therapeutic agents: II Virus reassortants map prevention of insulin-dependent diabetes mellitus to the small RNA of lymphocytic choriomeninitis virus. J Exp Med 1990; 171: 2091-2100 